\title{
Cave invertebrates in Espírito Santo state, Brazil: a primary analysis of endemism, threats and conservation priorities
}

\author{
Marconi Souza Silva', Rodrigo Lopes Ferreira ${ }^{2}$ \\ I Universidade Federal de Lavras, Departamento de Biologia, Setor de Zoologia Geral, Centro de Estudos em \\ Biologia Subterrânea \\ Corresponding author: Marconi Souza Silva (marconisilva@dbi.ufla.br)
}

Academic editor: Oana Moldovan | Received 1 May 2015 | Accepted 30 July 2015 | Published 14 August 2015

http://zoobank.org/AF2618A1-4E5C-4FC8-933E-EF3AE15D651F

Citation: Souza-Silva M, Ferreira RL (2015) Cave invertebrates in Espírito Santo state, Brazil: a primary analysis of endemism, threats and conservation priorities. Subterranean Biology 16: 79-102. doi: 10.3897/subtbiol.16.5227

\begin{abstract}
The cave-dwelling invertebrates were studied according to their composition, biodiversity, distribution and threats in the Atlantic Forest Central Biodiversity Corridor, a priority area for conservation actions in Brazil. Twelve obligate cave species were found, plus 495 troglophile species. Araneae (103 spp.), Coleoptera (61 spp.), Diptera (56 spp.) and Lepidoptera (38 spp.) were the richest taxa. The richness was higher in the carbonate caves (63 spp., sd $=16.7)$ and the highest diversity in granitic caves $\left(\mathrm{H}^{\prime}=2.68\right.$, $s d=0.5)$. The spatial turnover was 63.45 and similarity less than $30 \%$. The total richness was correlated with the linear extension of the caves ( $\mathrm{R} s=0.757, \mathrm{p} \leq 0.05)$. Surrounding area deforestation and religious and tourist use were the main threats. Emergency attention is recommended regarding protective actions, management and conservation of caves of extremely high biological importance.
\end{abstract}

\section{Keywords}

Atlantic forest, biodiversity, cave protection, subterranean habitats, troglobiont species

Copyright Marconi Souza-Silva, Rodrigo L. Ferreira. This is an open access article distributed under the terms of the Creative Commons Attribution License (CC BY 4.0), which permits unrestricted use, distribution, and reproduction in any medium, provided the original author and source are credited. 


\section{Introduction}

In the state of Espírito Santo, Brazil, the degradation of the Atlantic Forest has been as widespread and intense as in the rest of the country. From the original native forest cover, there is currently only approximately $11 \%$ remaining (Ribeiro et al. 2009, SOS Mata Atlântica and INPE 2014). Despite this fact, the state is still an important area of the Brazilian Atlantic Forest, in relation to the conservation of animal and plant biodiversity. Currently, there are regional planning geographical units in this state which comprise a mosaic of land uses and key conservation areas, established in workshops organized by Conservation International. Furthermore, Espírito Santo contains an area of the Brazilian Atlantic Forest Central Biodiversity Corridor (Aguiar et al. 2005, Galindo and Câmara 2005).

The corridor is extremely diverse, sheltering many animal and plant species with restricted distributions, including threatened species (Brown 1972, Thomaz and Monteiro 1997, Thomas et al. 1998, Passamani et al. 2000). As an example, the tableland forests of the state (Reserva Biológica de Sooretama and Reserva Florestal de Linhares) still harbor a diverse, but poorly known fauna (Peixoto and Gentry 1990, Chiarello 1995). Thus, there are still many knowledge gaps regarding the geography of the biodiversity in the state, especially due to the lack of published information for many groups. As an example, there are the natural subterranean cavities (caves) that, up to now, are represented in very few publications concerning inventories of their species (Ruschi 1952, Souza-Silva et al. 2011a, Souza and Ferreira 2011).

The first study regarding caves animals in the Atlantic Forest was made in 1907 in Iporanga municipality (São Paulo state), with the description of Pimelodella kronei (Ribeiro 1907), a troglobiont catfish species. However, descriptions of new invertebrate cave fauna species in this biome were only continued in the mid-1930's (CostaLima and Costa-Leite 1953); until the end of the 1970's the studies were still of a taxonomic nature and restricted to a few caves in the south of São Paulo state.

It was only in the 1980's that more general studies started to be conducted, when Dessen et al. (1980) published surveys of invertebrate cave fauna, some of them in the Brazilian Atlantic Forest. Subsequent studies were made in Ubajara municipality (Ceará state), southern Minas Gerais state, southern Bahia state (limestone province of Rio Pardo) and the Vale do Ribeira limestone province in the extreme south of São Paulo and northeast Paraná regions (Souza-Silva et al. 2015). Only after 2010 were geographically broader studies regarding the invertebrate cave fauna in the Brazilian Atlantic Forest conducted (CEBS 2015).

Caves are subterranean environments which harbor a rich fauna living with low food resource availability, constant temperatures and high humidity (Culver and $\mathrm{Pi}$ pan 2009, Souza-Silva et al. 2011b, Schneider et al. 2011). Many troglobiont cave species have restricted distributions and limited dispersal (Culver and Pipan 2009); consequently, they can be strongly affected by even moderate (human) changes in their environment. Furthermore, due to the high environmental stability of subterranean caves, such environments become highly susceptible to impacts, since this stable condition can often be easily unbalanced. Therefore, any direct or indirect impacts on 
caves can cause serious damage to the fauna and the physical integrity of these environments (Souza-Silva et al. 2015).

The characteristics most often mentioned to explain why some species are more vulnerable to extinction than others include small population sizes, low geographic distribution, specialized habitat requirements (Pimm et al. 1988, Purvis et al. 2000, Webb et al. 2002), large body sizes (McKinney 1997) and slow life histories (small broods, slow growth rates, late maturity, long inter-birth intervals, high survival rates) (MacArthur and Wilson 1967). Species may also become rare, and consequently vulnerable to extinction, because they are often hunted and may have their habitats destroyed and/or polluted (Whitmore and Sayer 1992, Bodmer et al. 1997, Isaac and Cowlishaw 2004).

Many troglobiont species have low population growth rates and in this case, strong environmental changes may increase the risk of extinction (Culver and Pipan 2009). The main threats come from the destruction of their habitats by mineral exploitation, tourism, vandalism, heavy metals, herbicide or pesticide pollution, eutrophication and biological invasions (Tercafs 1992, Ferreira and Horta 2001, Parise and Gunn 2007, Souza-Silva et al. 2015). Thus, many species that inhabit caves can be considered threatened and/or vulnerable to extinction due to habitat specificity that operates by selecting different ecological-evolutionary specializations (trogloxene, troglophile and troglobiont) to the hypogean conditions and environmental resources (Sket 2008).

The present study sought to evaluate the composition, richness, diversity, turnover and similarity of invertebrate cave fauna in the state of Espírito Santo, Brazil, an important geographical unit for conservation in the Atlantic Forest. Thus, we attempted to also evaluate the human changes to these caves, using a Cave Conservation Priority index (Souza-Silva et al. 2015), in order to suggest general conservation and management actions for the caves and their surrounding forest cover.

\section{Materials and methods}

\section{Study area}

The caves investigated in this study are located in the Atlantic Forest Central Biodiversity Corridor in Espírito Santo, Brazil, a priority area for conservation (Galindo and Câmara 2005). The rock types in which the sampled caves were developed were granite (11 caves), gneiss ( 1 cave), limestone (1 cave) and marble (2 caves) (Fig. 1 and Table 1 ).

\section{Sample data}

Only one visit to each cave was conducted and invertebrates present in the caves were collected manually in all existing potential biotopes and plotted on a schematic sketch of each cave (Souza-Silva et al. 2011a). Samples were collected with the aid of tweezers, brushes and entomological nets, prioritizing microhabitats such as driftwood, guano deposits, under rocks and damp locations. All organisms found were identified to the 
Table I. Location (UTM) and characteristics of 15 caves in the state of Espírito Santo, Brazil.

\begin{tabular}{l|l|l|l|l|l|l|l|l}
\hline Cave & Municipality & X & Y & L & A (m) & H & SE (m) & SV \\
\hline A. Pansini & Vargem Alta & 285168.01 & 7711062.66 & M & 450 & PS & 300 & M \\
\hline Casa Branca & Itaimbe-Itaguaçu & 305381.99 & 7830778.80 & Gr & 160 & DC & 15 & P \\
\hline Didi Vieira & Afonso Claúdio & 284809.28 & 7766144.11 & Gn & 662 & DC & 79 & SF \\
\hline Evald & Domingos Martins & 320007.63 & 7747606.91 & Gr & 705 & DC & 23 & M \\
\hline Faz. do Dr Saulo & Ecoporanga & 302473.87 & 7969940.28 & Gr & 223 & PS & 60 & P \\
\hline Faz. Paraíso & Ecoporanga & 306636.61 & 7957526.01 & Gr & 338 & DC & 13 & SF \\
\hline Henrique Altoé & Jaciguá & 289929.20 & 7709392.59 & Gr & 600 & PS & 90 & P \\
\hline Joáo Buteco & Ecoporanga & 308438.31 & 7974280.89 & Gr & 250 & DC & 25 & P \\
\hline Limoeiro & Conceição de Castelo & 273406.68 & 7733590.71 & C & 502.8 & DC & 600 & P \\
\hline Michele & Pancas & 311940.58 & 7872453.45 & Gr & 97 & PS & 60 & P \\
\hline Mirante & Vargem Alta & 285168.01 & 7711062.66 & M & 650 & PS & 30 & SF \\
\hline Represa & Santa Teresa & 322639.34 & 7808340.40 & Gr & 626 & PS & 25 & P \\
\hline Rio Itaúnas & Pedro Canário & 395452.20 & 7977430.06 & Gr & 301 & PS & 41 & P \\
\hline Ruschi & Santa Tereza & 339370.99 & 7791692.36 & Gr & 672 & TS & 30 & SF \\
\hline Santa Bárbara & Venda N. Imigrantes & 275936.82 & 7747596.29 & Gr & 1116 & WT & 80 & M \\
\hline
\end{tabular}

(L) lithology, (A) altitude, (H) hydrology, (SE) sampled linear extension, (SV) 250m surrounding vegetation, (DC) dry cave, (TS) temporary stream, (WT) water table, (PS) perennial stream (Gr) granite (Gn) gneiss, $(\mathrm{M})$ marble and $(\mathrm{C})$ calcareous, $(\mathrm{SF})$ secondary forest, $(\mathrm{P})$ pasture and $(\mathrm{M})$ monoculture.

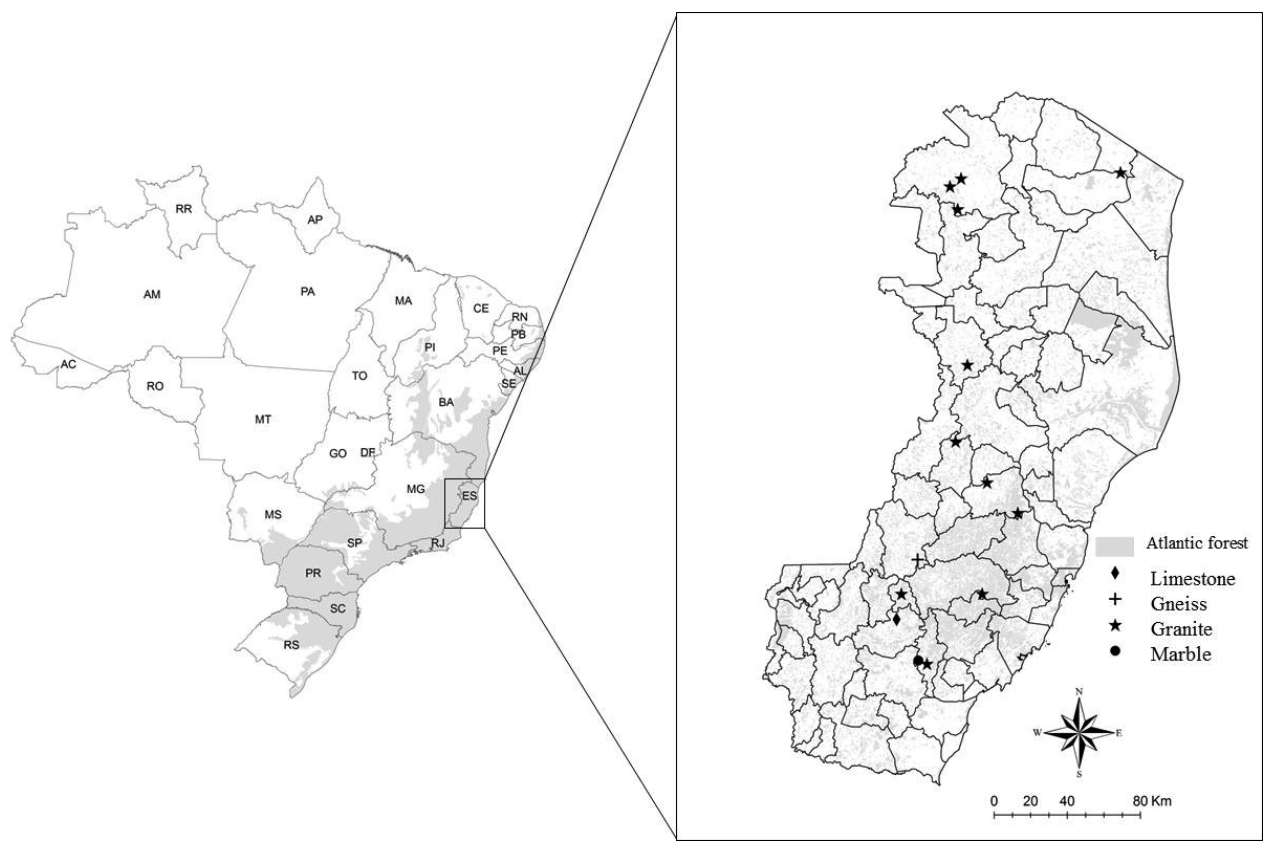

Figure I. Distribution of 15 caves in the Atlantic Forest in the state of Espírito Santo, with invertebrate fauna inventoried in this study. Source: SOS Mata Atlântica (2011). 
accessible taxonomic level and grouped into morphotypes (Souza-Silva et al 2011a). The trophic characterization of caves was conducted concurrently with the invertebrate collections. For such, all organic resources present were noted and when possible their pathways to cavities characterized. The quality or quantity of food was not calculated and analyzes of water and soil nutrients were also not carried out.

Uses and environmental changes (impacts) in the caves and surroundings were evaluated based on forms filled out during visits (Simões et al. 2014, Souza-Silva et al. 2015).

\section{Cave invertebrate community structure}

To standardize the richness values used in the comparisons of caves, they were relativized according to the linear development and horizontal extension of the entrances of each cave (biological variable/cave linear development $/ \Sigma$ entry width) (Souza Silva et al. 2011a). The relative richness categorizes the number of species as a function of the cave length and the extension of the entrances. Thus, this variable seeks to reduce excessive contribution of the para-epigean community by considering the entrance extension in this analysis. It is expected that in the caves with extended entrances there is a great contribution by the para-epigean communities due to increased contact with the external environment (Prous et al. 2004, Prous et al. 2015).

\section{Statistical analysis}

The alpha diversity values $(\alpha)$ of invertebrate communities associated with each cave were calculated using the Shannon-Weaver index (Magurran 2004). The beta diversity ( $\beta$ or turnover) was calculated, using the presence and absence of data, by the Harrison ratio (1992). $\beta_{\text {Harrison }}=\{[(S / \alpha)-1] /(\mathrm{N}-1)\}^{*} 100$, where $S=$ total number of species, $\alpha=$ average richness and $\mathrm{N}=$ number of samples. This measure ranges from 0 (no turnover) to 100 (each sample has a single set of species) (Magurran 2004). The Berger-Parker dominance index was used for assessing the relative importance of dominant species in the communities (Magurran 2004). The similarities among the cave fauna were obtained through the Bray-Curtys index (Magurran 2004). The Spearman correlation (Rs) was used to detect possible relationships between total species richness and cave horizontal projection and entrance extension variables. Possible differences between richness and relative and total diversity among caves in carbonate (limestone and marble) and granitic rock (granite and gneiss) were evaluated using the Kruskal-Wallis test (Zar 1984). The software used for the analysis was PAST (Hammer et al. 2001).

\section{Cave Conservation Priorities}

To assess the status of threats to biodiversity in caves of the Atlantic Forest in Espírito Santo, we used the Cave Conservation Priority index (CCP $i$ ) proposed by Souza-Silva 
et al. (2015). The CCP $i$ provides a vulnerability score for each cave using the overlap of biological relevance (BR) and human impact degree (HID) (Souza-Silva et al. 2015). The biological relevance of the caves can be determined through the superimposition of three variables: troglobiont species richness $\left(\mathrm{T}_{\mathrm{g} / \mathrm{b}} \mathrm{R}\right)$, total and relative troglophile richness $\left(T_{t f} R\right.$ and $\left.R_{t r} R\right)$. For each of these variables categories as weights were used, extremely high (weight 4), high (weight 3), average (weight 2) and low (weight 1) to facilitate valuations. Based on the highest sum value for these weights (8) $\left(\mathrm{BR}_{\text {weight }}+\mathrm{HID}_{\text {weight }}\right)$ four cave vulnerability or conservation priority categories were created. Such categories include extremely high (weight $\geq 6$ ), high (4-5.99) average ( $2-4.99)$, and low ( $\leq 1.99)$ (Souza-Silva et al. 2015).

\section{Human impacts}

The human modifications surveyed in this study were classified in relation to uses and impacts. Tourist and religious activities were considered uses, impacts being trampling, illumination and construction resulting from these activities (Souza-Silva et al. 2015).

Environmental changes (impacts) were defined for each cave in function of the presence or absence of modifications inside and in the surroundings. Surroundings was considered as a 250 meter radius projected from the cave's external contour, according to Brazilian law (CONAMA no 347 , September, $10^{\text {th }}$, 2004, Souza-Silva et al. 2015). The impacts were considered as those modifications that could potentially lead to depletion (a), enrichment (b) or alteration (c) in the microhabitats, organic resources and/or cave fauna (Souza-Silva et al. 2015).

The urgent conservation and management actions for the caves and their surrounding forest cover were based on six criteria: (1) microbiological research (suggested for caves with probable occurrence of human pathogenic fungi), (2) defining the abundance of troglobiont species, (3) recovery of the surroundings (suggested for caves with deforested surroundings area), (4) management plan (suggested for caves with intense human use), (5) maintenance of the surroundings using Private Natural Heritage Reserves-PNHRs, (suggested for caves with preserved surroundings area or in advanced succession), (6) compensatory measures (suggested for caves made completely uncharacteristic as a result of human activities).

Private Natural Heritage Reserves (PNHRs) is a category in the current National Conservation Unit System (Law 9.985 of 2000 - SNUC) that supports the creation of protected areas of variable sizes and uses (Maciel 2000).

\section{Karstic areas for conservation priorities}

The results of the CCPi associated with troglomorphic traits and troglobiont species richness and distribution were used to indicate karst areas that deserve attention with respect to conservation needs. 


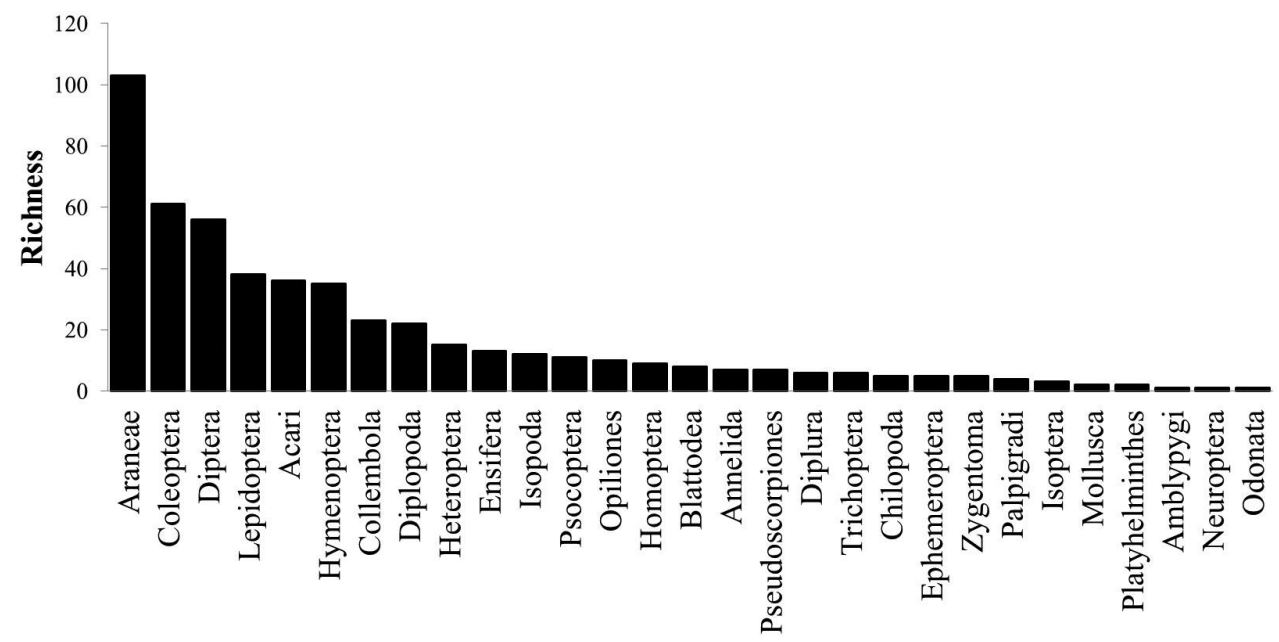

Figure 2. Composition and richness of invertebrate taxa collected in 15 caves in the state of Espírito Santo, Brazil.

\section{Results}

\section{Invertebrate diversity and distribution}

507 invertebrate species were collected, distributed in at least 121 families (Table 2). The taxa Acari (36 spp.), Araneae (103 spp.), Opiliones (10 spp.), Diplopoda (22 spp.), Collembola (23 spp.), Coleoptera (61 spp.), Diptera (56 spp.), Lepidoptera (38 spp.), Hymenoptera (35 spp.), Heteroptera (15 spp.), Ensifera (13 spp.), Isopoda (12 spp.) and Psocoptera (11 spp.) were the richest (Fig. 2).

Twelve species with troglomorphic traits were found, all arthropods, with distributions restricted to six caves: one isopod (Trachelipodidae) and one spider (Ochiroceratidae) both from a granitic cave, in Santa Teresa municipality; a silverfish (Zygentoma: Lepismatidae) and an isopod (Isopoda: Plathyarthridade: Trichorhina sp.) both from a granitic cave in Ecoporanga municipality; another silverfish species (Zygentoma: Lepismatidae) from a granitic cave in Pedro Canário municipality; a palpigrade (Eukoeneniidae: Eukoenenia spelunca, Souza and Ferreira 2011), a harvestman (Opiliones: Escadabiidae) and a millipede (Polydesmida; Trichoplydesmidae) from a granitic cave in Jaciguá municipality and two millipedes (Spirostreptida: Pseudonannolenidae: Pseudonannolene sp) and a Polydesmida: Cryptodesmidae from a marble cave in Vargem Alta municipality and another harvestman (Opiliones: Escadabiidae) from a carbonate cave in Conceição do Castelo municipality (Table 3). Most of the troglomorphic specimens collected were sent to specialists and are under description (Fig. 3).

The total richness varied between 17 to 79 species, and the average species richness was 51 species. The relative richness varied between 0.01 to 1.03 , and the average relative species richness was 0.25 (Figures 4 and 5). The total abundance varied from 50 to 9,029 individuals per cave and the average abundance was 1,421 individuals. The dominance varied from 0.06 to 2 and the average dominance was 0.13 . The diversity 
Table 2. Composition and distribution of invertebrate taxa observed in 15 caves in the state of Espírito Santo, Brazil. Legend: Didi Vieira (DV), Fazenda Paraíso (FP), Michele (Mi), Limoeiro (L), Represa (RE), João Buteco (JB), Casa Branca (CB), Ruschi (RU), Evald (E), Santa Barbára (SB), Fazenda do Dr Saulo (FS), Mirante (Mr), Archimedes Pasinsi (AP), Henrique Atoé (HA), Rio Itaúnas (RI).

\begin{tabular}{|c|c|c|c|c|c|c|c|c|c|c|c|c|c|c|c|c|}
\hline Taxa & Family/subfamily & DV & FP & Mi & $\mathbf{L}$ & RE & JB & $\mathrm{CB}$ & $\mathbf{R u}$ & $\mathbf{E}$ & SB & FS & Mi & $\mathbf{A P}$ & HA & RI \\
\hline Turbellaria & Dugesiidae & & & + & & & & & & & & + & & & & \\
\hline Oligochaeta & unidentified & + & + & + & + & + & & & + & & & + & & + & + & \\
\hline \multirow{5}{*}{ Isopoda } & unidentified & & & + & & + & & & & & & + & & & & \\
\hline & Trachelipodidae & & & & & & & & + & & & & & & & \\
\hline & Philosciidae & & & & & + & & & + & & & & & & & \\
\hline & Platyarthridae & & & + & + & & & & & & & + & & + & + & + \\
\hline & Armadillididae & & & & & & & + & & & & & & & & \\
\hline \multirow{15}{*}{ Acari } & unidentified & + & & + & + & & & + & + & & + & + & + & + & + & + \\
\hline & Acaridae & & & & & & & & & & & & + & & & \\
\hline & Anystidae & + & & & & & & & & & & & & & & \\
\hline & Argasidae & & + & & & + & & & & & + & & & + & + & \\
\hline & Ascidae & & & & & + & & & & & & & & & & \\
\hline & Bdellidae & & & + & & & & + & + & & + & & & & & \\
\hline & Cunaxidae & & & & & & & & + & & & & & & & \\
\hline & Erythraeidae & & & + & & & & + & + & & & + & & & + & \\
\hline & Labidostomatidae & & & & + & & & & & & & & & & & \\
\hline & Laelapidae & & & & + & & & & + & & & & & + & & \\
\hline & Macrochelidae & & & & & & & & & & & & + & & & \\
\hline & Macronyssidae & & & + & & & & & & & & + & & & & \\
\hline & Podocinidae & & & + & & & & & & & & & & & & \\
\hline & Trombidiidae & & & & & & & & + & & & & + & + & & \\
\hline & Veigaiidae & & & + & & & & & + & & & & & & & \\
\hline Amblypygi & Charinidae & & & & & + & & & & & & & & & & \\
\hline \multirow{19}{*}{ Araneae } & unidentified & + & & & + & + & & & + & + & + & + & & + & + & \\
\hline & Araneidae & + & & & & & + & & & + & & & & + & & \\
\hline & \begin{tabular}{|l|} 
Clubionidae \\
\end{tabular} & + & & & + & & & & & & & + & & + & & \\
\hline & \begin{tabular}{|l|} 
Ctenidae \\
\end{tabular} & + & + & + & + & & & & & + & + & + & + & + & + & + \\
\hline & Dipluridae & + & + & & & & & + & + & & & + & & & & + \\
\hline & Dysderidae & & & & & & & & & & & & & & & + \\
\hline & Gnaphosidae & & + & & & & & & + & & & & & & + & \\
\hline & Lycosidae & & & + & & & & & & & & & & & & \\
\hline & Lyniphidae & & & & + & & & & & & & & & & & \\
\hline & Nemesidae & & & & + & & & & & & & & & & & \\
\hline & Ochiroceratidae & & & & & & & & + & & & & & & & \\
\hline & Oecobidae & & & & + & & & & & & & & & & & \\
\hline & Oonopidae & + & & + & + & + & & + & + & & & & & + & + & + \\
\hline & Pholcidae & & + & + & + & + & & + & + & + & + & + & + & + & + & + \\
\hline & Salticidae & + & + & & + & & + & & & & & + & & & & \\
\hline & Scicariidae & & & & + & & & & & & & & + & + & & \\
\hline & Scytodidae & & & + & + & + & + & + & & & & & & & & + \\
\hline & Tetragnathidae & & & & + & & & + & & & & & & & & \\
\hline & Theraphosidae & & & & & + & & & & & & & & & & \\
\hline
\end{tabular}




\begin{tabular}{|c|c|c|c|c|c|c|c|c|c|c|c|c|c|c|c|c|}
\hline Taxa & Family/subfamily & DV & FP & Mi & $\mathbf{L}$ & RE & JB & CB & $\mathbf{R u}$ & $\mathbf{E}$ & SB & FS & Mi & $\mathbf{A P}$ & HA & RI \\
\hline & Theridiidae & + & & & + & + & + & + & + & + & + & + & & & + & + \\
\hline & Theridiosomatidae & & + & + & & & & & & & & + & + & + & + & \\
\hline & Uloboridae & & & & & & & + & + & & + & & & & & \\
\hline \multirow{2}{*}{ Opiliones } & Gonyleptidae & & + & & + & & & & + & & + & & + & + & + & \\
\hline & Escadabiidae & & & & & & & & & & & & & + & & \\
\hline Palpigradi & Eukeneniidae & + & & + & & & & & + & & & & & + & & \\
\hline Pseudoscorpiones & Chernetidae & + & + & + & & & & & + & & + & & & + & + & + \\
\hline \multirow{5}{*}{ Diplopoda } & unidentified & & & & & & & & & & & & & & + & \\
\hline & Cryptodesmidae & + & + & + & & & & & & & & & & + & & \\
\hline & Pseudonannolenidae & & & + & + & & & & & & + & & & + & + & \\
\hline & Pyrgodesmidae & & & & & & & & + & & + & & & + & & + \\
\hline & Rinocricidae & & & & + & & & & & & & & & & & \\
\hline \multirow{2}{*}{ Polydesmida } & unidentified & & & & & + & & & & & + & & & & + & \\
\hline & Trichopolydesmidae & & & & & & & & & & & & & & & + \\
\hline Spirobolida & unidentified & & & & + & & & + & & & & & & & + & \\
\hline Geophilomorpha & unidentified & + & & & & + & & & & & & & & & & \\
\hline Litobiomorpha & unidentified & & & & & & & & + & & & & & & & \\
\hline Polyxenida & unidentified & & & & + & & & & & & & & & & & \\
\hline Symphyla & unidentified & + & + & + & & + & & & + & & + & + & & + & & \\
\hline Archaeognatha & Meinertellidae & & & + & & & & & & & & & & & & \\
\hline Blattodea & unidentified & & & + & + & & + & & + & & + & + & + & + & & + \\
\hline \multirow{15}{*}{ Coleoptera } & unidentified & + & + & + & + & & + & & + & & + & & + & & & + \\
\hline & Carabidae & + & & + & & & & & + & & + & & + & & & \\
\hline & Chrysomelidae & & & & + & & & & & & & & & & & \\
\hline & Dermestidae & + & & & & & & & & & & & & & & \\
\hline & Dryopidae & & & + & & & & & & & & & & & & \\
\hline & Elateridae & & & & + & & & & & & & & & & & \\
\hline & Elmidae & & & & & & & & & & & & & + & & \\
\hline & Histeridae & & & + & & & + & & & & & & & + & + & + \\
\hline & Lampyridae & & & & & & & & + & & + & & & & & \\
\hline & \begin{tabular}{|l|} 
Leiodidae \\
\end{tabular} & & & & & & & & & & + & & & & & \\
\hline & Cholevidae & & & & & & & & & & + & & & & & \\
\hline & Pselaphidae & + & + & + & & & & & + & & + & & & + & + & \\
\hline & Scarabeidae & & & & & & & & + & & & & & & & \\
\hline & Staphylinidae & & + & + & + & + & & & + & + & + & + & + & & + & + \\
\hline & Tenebrionidae & + & + & + & + & & + & & & + & & + & & + & & + \\
\hline Collembola & unidentified & + & + & + & + & + & & & + & & + & + & + & + & + & + \\
\hline \multirow{4}{*}{ Diplura } & Anajapygidae & & & & & & & & + & & & & & & & \\
\hline & Campodeidae & + & + & & & & & & + & & & & & & & \\
\hline & Japygidae & & & & & & & & + & & & & & & & \\
\hline & Projapygidae & + & + & & & & & & & & & & & & & \\
\hline \multirow{5}{*}{ Diptera } & unidentified & + & & + & + & & + & & & & + & & + & + & & + \\
\hline & Agromyzidae & & & + & + & & & & + & + & + & & & + & & \\
\hline & Calliphoridae & & & & & & & & + & & & & & + & & \\
\hline & Cecidomyidae & & & & & & & + & & & & & & & & \\
\hline & Chironomiidae & & & & & & & & & & & & & + & & \\
\hline
\end{tabular}




\begin{tabular}{|c|c|c|c|c|c|c|c|c|c|c|c|c|c|c|c|c|}
\hline Taxa & Family/subfamily & DV & FP & Mi & $\mathbf{L}$ & $\mathbf{R E}$ & JB & CB & $\mathbf{R u}$ & $\mathbf{E}$ & SB & FS & Mi & AP & HA & RI \\
\hline & Culicidae & + & & & & & & + & & & + & & & & + & \\
\hline & Dixidae & & & & + & & & & & & + & & + & + & & \\
\hline & Dolychopodidae & + & & & + & & & & & & & & + & & & \\
\hline & Drosophilidae & & & & & & + & + & & & & + & + & & & + \\
\hline & Keroplatidae & & + & & & & & & & & & & + & & & + \\
\hline & Lauxanidae & & & & + & & & & & & + & & & & + & \\
\hline & Lonchaeidae & & & & & & + & & & & & & & & & \\
\hline & Milichidae & & & & & & & & & & & & & & & + \\
\hline & Mycetophilidae & & & & & & & & & & & & & & + & \\
\hline & Nematocera & + & & & + & + & & & + & & + & + & + & & & \\
\hline & Phoridae & & + & & & + & & & + & + & + & + & + & + & + & \\
\hline & Psychodidae & & + & + & + & & + & & + & & & + & + & & + & + \\
\hline & Sciaridae & + & & + & + & + & & & & & & + & & & & \\
\hline & Simulidae & & & + & & & & & & & & & + & + & & \\
\hline & Sphaeroceridae & & & & + & & & & & & & & & & & \\
\hline & Empididae & & & + & & & & & & & & & & & & \\
\hline & Syrphidae & + & & & & + & & & & & & & & & & \\
\hline & Tipulidae & + & & & & & & & + & & & + & & & & \\
\hline \multirow{2}{*}{ Ensifera } & Phalangopsidae & & + & + & + & + & & + & + & + & + & + & + & + & + & + \\
\hline & Tetigoniidae & + & & & & & & & & & & & & & & + \\
\hline Ephemeroptera & unidentified & & & + & & & & & & & & & + & + & & + \\
\hline \multirow{7}{*}{$\begin{array}{l}\text { Hemiptera/ } \\
\text { Heteroptera }\end{array}$} & unidentified & & & + & & & & & & & & & & & & + \\
\hline & Cydnidae & & + & & & 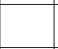 & & & + & & + & + & & & + & + \\
\hline & Gelastocoridae & & & & & + & & & & & & & & & & \\
\hline & Naucoridae & & & + & & & & & & & & & & & & \\
\hline & Emesiinae & + & + & & + & & & & & + & & & + & + & & + \\
\hline & Reduviinae & + & + & + & + & + & & & + & + & + & + & + & + & + & \\
\hline & Vellidae & & & & & & & & & & + & + & & + & & \\
\hline \multirow{3}{*}{$\begin{array}{l}\text { Hemiptera/ } \\
\text { Homoptera }\end{array}$} & unidentified & & & + & & & & & & & & & & & & \\
\hline & Cixidae & + & + & + & & + & & & + & & & & & & & \\
\hline & Membracidae & + & & & & & & & & & + & & & & & \\
\hline \multirow{8}{*}{ Hymenoptera } & unidentified & + & + & + & + & & & & + & & + & & & + & + & \\
\hline & Brachonidae & & & & & & & & & & + & & & & & + \\
\hline & Evaniidae & & & & + & & & & & & & & & & & \\
\hline & Formicidae & + & + & + & + & + & + & + & + & + & + & + & + & & + & + \\
\hline & Halictidae & + & & & & & & & & & & & & & & \\
\hline & Mutilidae & & & & + & & & & & & & & & & & \\
\hline & Sphecidae & + & & & & & & & & & & + & & & & + \\
\hline & Vespidae & & & & + & & & & & & & & & & & \\
\hline Isoptera & Termitidae & + & & + & & + & & & & & & & + & + & + & \\
\hline \multirow{4}{*}{ Lepidoptera } & unidentified & + & & & + & & & + & & & + & + & & + & & \\
\hline & Geometridae & & & + & & & & & & & & & & & & \\
\hline & Hesperiidae & + & & & & & & & & & & + & & & & \\
\hline & Noctuidae & + & & + & & & + & + & & & & & & + & & \\
\hline
\end{tabular}




\begin{tabular}{|c|c|c|c|c|c|c|c|c|c|c|c|c|c|c|c|c|}
\hline Taxa & Family/subfamily & DV & FP & Mi & $\mathbf{L}$ & RE & JB & CB & $\mathbf{R u}$ & $\mathbf{E}$ & SB & FS & Mi & AP & HA & RI \\
\hline & Nymphalidae & + & & & & & & & & & & & & & & \\
\hline & Tineidae & + & + & + & + & + & & + & + & + & & + & + & + & + & + \\
\hline Neuroptera & Myrmeleontidae & + & & & + & & & & & & & & + & & & \\
\hline Odonata & unidentified & & & + & & & & & & & & & & & & \\
\hline \multirow{4}{*}{ Psocoptera } & unidentified & & & & & & & & + & & + & & & & & \\
\hline & Lepidopsoscidae & & & & & & & & & & + & & & & & \\
\hline & Pseudocaeciliidae & & & & & & & & + & & & & & & & \\
\hline & Psyllipsoscidae & & & & + & & & & & & & & + & + & & \\
\hline \multirow{2}{*}{ Trichoptera } & unidentified & & & & & + & & & & & & & + & & & \\
\hline & Hydropschidae & & & & & & & & & & & + & + & & & \\
\hline \multirow{2}{*}{ Thysanura } & Lepidotrichidae & & & & & & & & + & & & & & & & \\
\hline & Lepismatidae & & & & & & + & & & & & + & & & & + \\
\hline Mollusca & unidentified & & & + & & & & & & & & & & & & \\
\hline
\end{tabular}

Table 3. Biological characteristics and priority actions for 15 caves in the state of Espírito Santo, Brazil. Legend: (S) total richness (A) abundance, (D) dominance, $(\mathrm{H})$ diversity, (E) equitability, (TR) organic resources (HG) hematophagous guano (PD) plant debris (CG) carnivore guano, (FG) frugivore guano (R) roots. (PA) priority actions (1) microbiological research, (2) define status of endemic species, (3) recovery of the surroundings, (4) management plan, (5) maintenance of the surroundings, (6) compensatory measure. (T) troglomorphic taxa.

\begin{tabular}{c|c|c|c|c|c|c|c|c}
\hline Cave & S & A & D & H & E & TR & PA & T \\
\hline Archimedes Pansini & 66 & 760 & 0.08 & 2.99 & 0.71 & HG & 2,3 & $\begin{array}{c}\text { Eukoenenia spelunca, Pseudonnanolene } \\
\text { sp. n., Cryptodesmidae sp. n., } \\
\text { Escadabiidae sp. n., Trichorhina sp. n. }\end{array}$ \\
\hline Casa Branca & 41 & 183 & 0.2 & 2.36 & 0.64 & $?$ & 6 & \\
\hline Didi Vieira & 64 & 231 & 0.06 & 3.51 & 0.84 & PD & 5 & \\
\hline Evald & 17 & 50 & 0.19 & 2.23 & 0.79 & PD & 3 & \\
\hline Fazenda do Dr. Saulo & 46 & 1162 & 0.11 & 2.61 & 0.68 & HG & 2,3 & Zygentoma and Trichorhina sp. n. \\
\hline Fazenda Paraiso* & 40 & 797 & 0.18 & 2.12 & 0.57 & CG & 1 & Trichopolydesmidae sp. n. \\
\hline Henrique Altoé & 50 & 854 & 0.14 & 2.44 & 0.63 & R, HG & 3,4 & Opiliones \\
\hline Joáo Buteco & 17 & 646 & 0.16 & 2.17 & 0.76 & HG & 3 & \\
\hline Limoeiro & 78 & 4074 & 0.20 & 2.38 & 0.54 & HG & $2,3,4$ & \\
\hline Michele & 73 & 245 & 0.06 & 3.48 & 0.81 & R, HG & 3 & \\
\hline Mirante & 45 & 1920 & 0.2 & 2.11 & 0.55 & HG & 5 & \\
\hline Represa & 43 & 297 & 0.1 & 2.86 & 0.76 & R & 3,5 & \\
\hline Rio Itaúnas & 49 & 9029 & 0.12 & 2.28 & 0.59 & FG & 2,3 & Zygentoma and Trichorhina sp. n. \\
\hline Ruschi & 79 & 462 & 0.09 & 3.13 & 0.72 & PD; HG & 2,5 & Trachelipodidade and Araneae \\
\hline Santa Bárbara & 61 & 617 & 0.08 & 2.95 & 0.72 & HG & $3,4,5$ & \\
\hline Average & 51.3 & 1421 & 0.13 & 2.64 & 0.60 & & & \\
\hline Standard Deviation & 19 & 2330 & 0.05 & 0.48 & 0.1 & & & \\
\hline
\end{tabular}

* Probable occurrence of strains of Histoplasma sp. associated with the guano. Two team members collecting fauna had contact with guano, presented symptoms and diagnosed positive for Histoplasmosis. Farm owner reported that a nephew who had visited the caves showed Histoplasmosis symptoms, but without a confirmed diagnosis. 


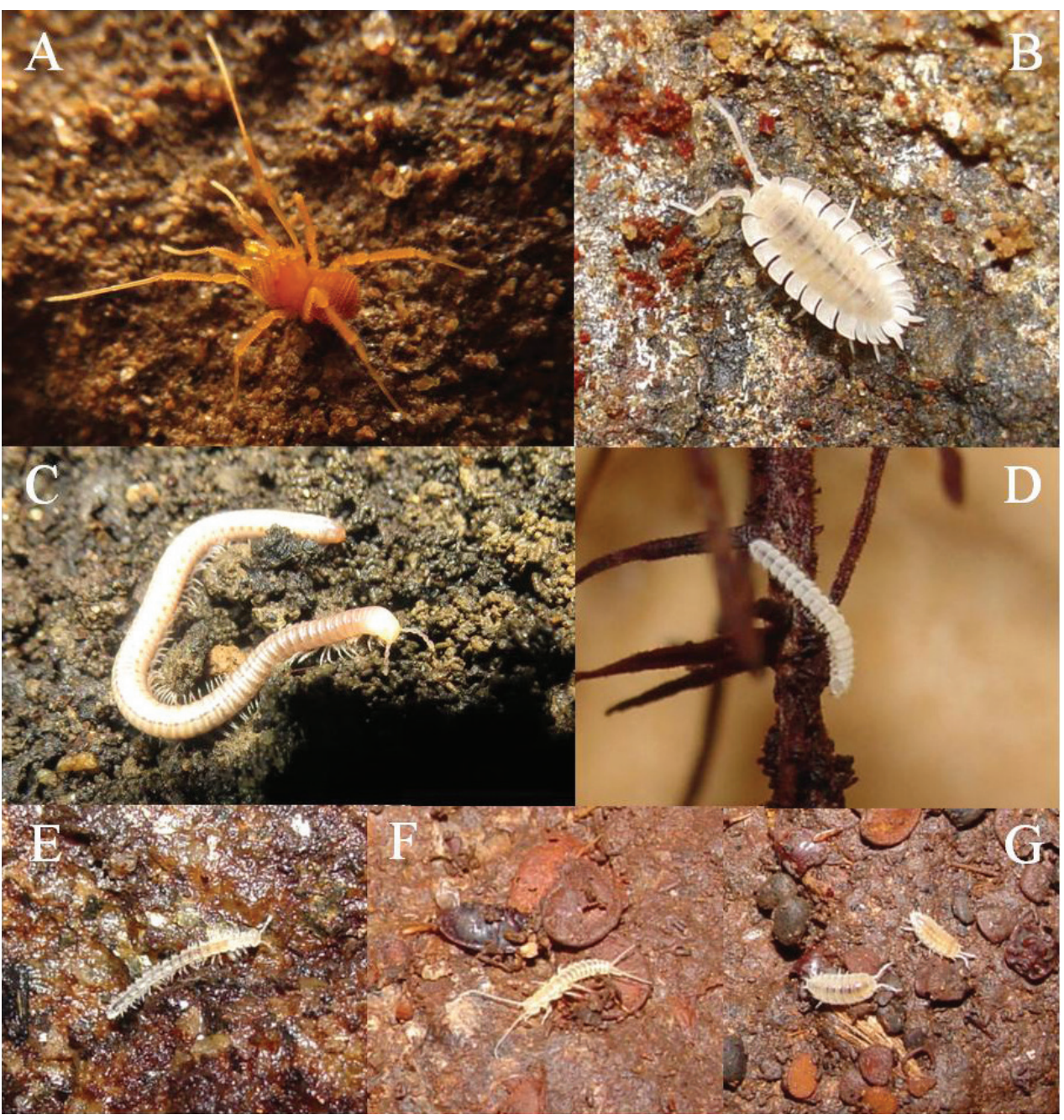

Figure 3. Some of the troglomorphic invertebrates sampled in 15 caves in Atlantic forest at Espírito Santo state, Brazil. A Escadabiidae, B Trachelipodidae, C Pseudonannolene sp., D Cryptodesmidae, E Trichopolidesmydae, F Zygentoma, G Trichorbina sp.

varied between 2.11 to 3.51, and the average diversity was 2.64 (Table 3 and Fig. 4). The $\beta$ diversity was 63.45 . The similarity of the fauna was low, less than $30 \%$. The total richness correlated positively and significantly $\left(R_{s}=0.757\right)$ with the linear extension of the caves (Fig. 6A). However, when the carbonate caves were excluded from the sample, there was no significance in the test (Fig. 6B).

The average richness was higher in the limestone caves (marble and calcareous) (63 spp., sd $=16.7)$ and the diversity was higher in the granite caves $\left(H^{\prime}=2.68\right.$, sd $=$ $0.5)$, but there were no significant differences in average richness and diversity between carbonate and granite caves (Fig. 7). 


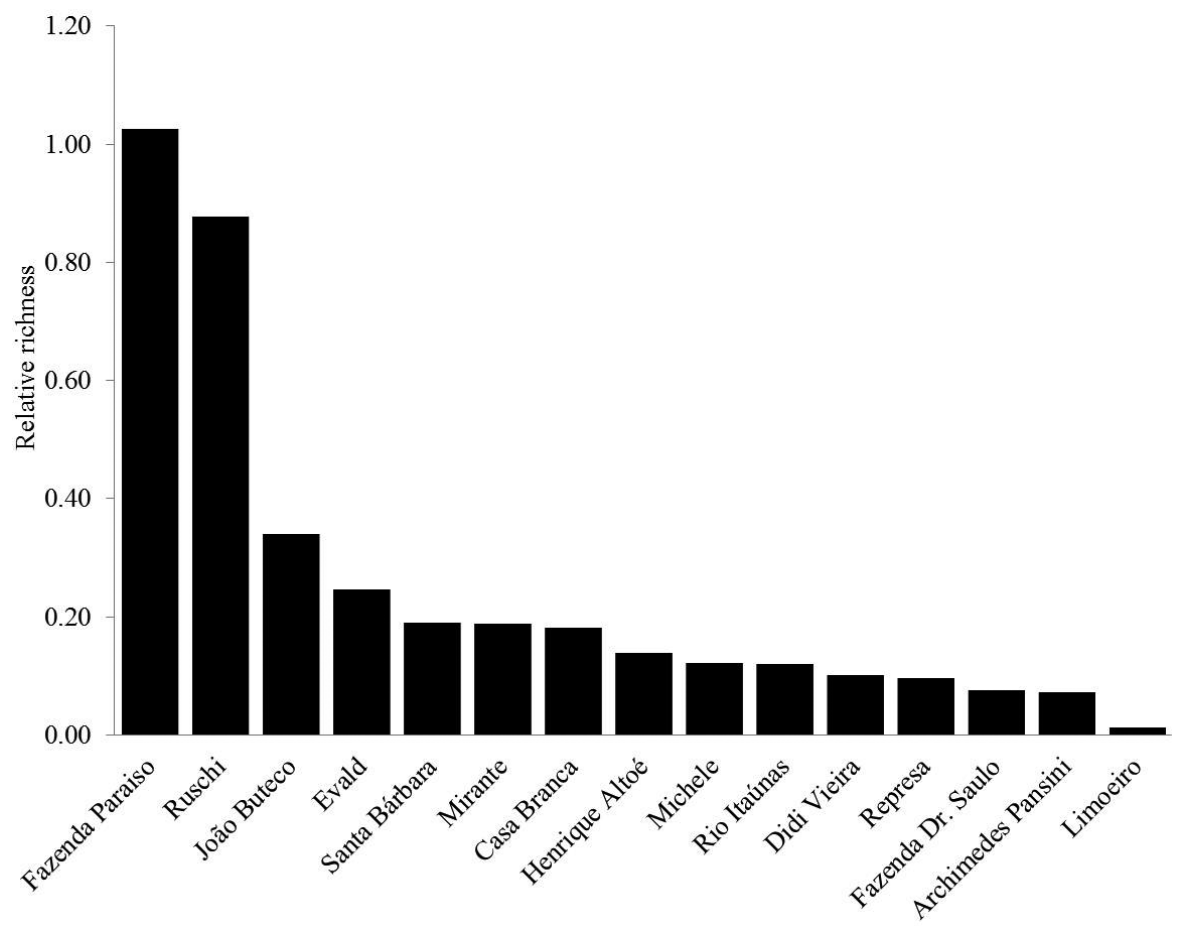

Figure 4. Relative richness of the invertebrate fauna in 15 caves in the state of Espírito Santo.

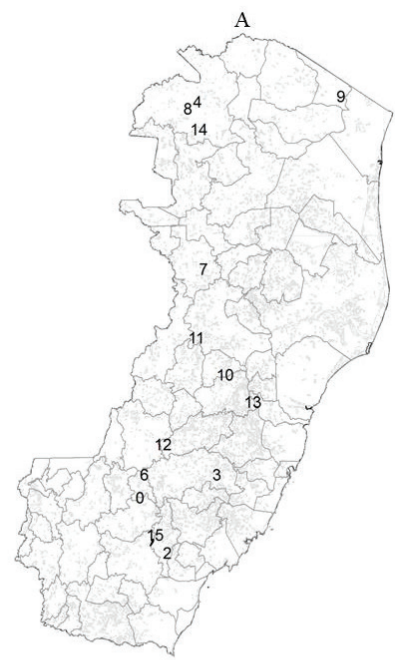

(0) Limoeiro, (1), Archimides Pansini, (2) Henrique atoé, (3) Evald, (4) João Buteco, (5) Mirante, (6) Santa Barbara, (7) Michele, (9), Gruta do Rio Itaúnas, (8) Fazenda do Dr. Saulo, (10), Gruta da represa, (11) Casa branca, (12) Didi Vieira, (13) Gruta do Ruschi, (14) Fazenda Paraiso.

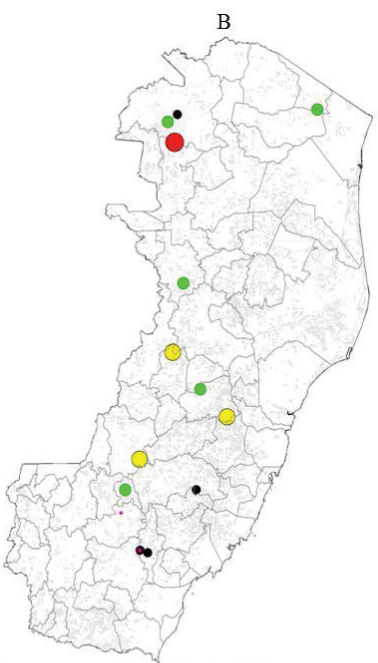

$0.25-0.44$

- $0.441-0.94$

$0.941-1.466$

$1.461-2.11$

$2.111-3.07$

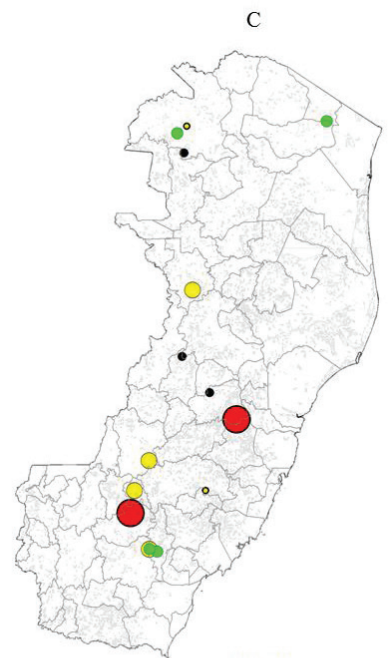

- $17-18$

- 18.1- 44

(1) 44.1 - 51

○ $51.1-70$.

$70.1-77$

Figure 5. A Distribution of caves, $\mathbf{B}$ relative richness and $\mathbf{C}$ total richness of the 15 caves of the state of Espírito Santo, Brazil. 
Table 4. Qualification of use occurrence * and human alteration weights in 15 caves in the state of Espírito Santo, Brazil.

\begin{tabular}{l|c|c|c|c|c|c|c|c|c|c|c|c}
\hline Cave & $\mathbf{1}^{*}$ & $\mathbf{2}^{*}$ & $\mathbf{3}$ & $\mathbf{4}$ & $\mathbf{5}$ & $\mathbf{6}$ & $\mathbf{7}$ & $\mathbf{8}$ & $\mathbf{9}$ & $\mathbf{1 0}$ & Sum & IW \\
\hline Archimedes Pansini & & + & 1 & & 7 & & 7 & & & 3 & 18 & $\mathrm{H}$ \\
\hline Casa Branca & + & & 1 & & 7 & 4 & 7 & & 7 & & 26 & $\mathrm{EH}$ \\
\hline Didi Vieira & & & & & & & & & & & 0 & $\mathrm{~L}$ \\
\hline Evald & & & 1 & & & & & & & & 1 & $\mathrm{~L}$ \\
\hline Fazenda do Dr. Saulo & & & 1 & & & & & 6 & & & 7 & $\mathrm{~A}$ \\
\hline Fazenda Paraíso & & & & & & & & & & & 0 & $\mathrm{~L}$ \\
\hline Henrique Atoé & & & 1 & & & & & & & & 1 & $\mathrm{~L}$ \\
\hline João Buteco & & & 1 & & & & 7 & & & & 8 & $\mathrm{~L}$ \\
\hline Limoeiro & + & + & 1 & 4 & 7 & 4 & 7 & & 7 & 3 & 33 & $\mathrm{EH}$ \\
\hline Michele & & & 1 & & & & & & & & 1 & $\mathrm{~L}$ \\
\hline Mirante & & & & & & & & & & & 0 & $\mathrm{~L}$ \\
\hline Represa & & & 1 & & & & & & & & 1 & $\mathrm{~L}$ \\
\hline Rio Itaúnas & & & & & & & & & & & 0 & $\mathrm{~L}$ \\
\hline Ruschi & + & + & 1 & & 7 & 4 & 7 & & & & 19 & $\mathrm{H}$ \\
\hline Santa Bárbara & & & & & & & & & & & & \\
\hline
\end{tabular}

Legend: Religious use (1), tourist use (2), surrounding deforestation up to $250 \mathrm{~m}$ (3), detonations inside (4), trampling inside (5), construction inside (6), trash inside (7), subterranean drainage exploitation (8), electric lighting (9) depredation of speleothems (10). (IW) impact weight categories. Extremely high (EH), High (H), Average (A), Low (L).

Table 5. Categorization of the caves regarding biological relevance, impact degree and vulnerability of their communities. Caves with troglomorphic species $\left({ }^{*}\right)$.

\begin{tabular}{c|c|c|c|c}
\hline Cave & $\begin{array}{c}\text { Troglomorphic } \\
\text { relevance }\end{array}$ & $\begin{array}{c}\text { Biological } \\
\text { relevance }\end{array}$ & Impacts degree & $\begin{array}{c}\text { Vulnerability to } \\
\text { protect }\end{array}$ \\
\hline Didi Vieira & Low & High & Low & Average \\
\hline Evald & Low & Low & Low & Average \\
\hline Faz. Paraíso & Low & High & Low & Average \\
\hline Henrique Atoé & Low & Average & Low & Average \\
\hline Joáo Buteco & Low & Average & Low & Average \\
\hline Michele & Low & High & Low & Average \\
\hline Mirante & Low & High & Low & Average \\
\hline Represa & Low & Average & Low & Average \\
\hline A. Pansini & Extremely High & Extremely high & High & Extremely high \\
\hline Faz. do Dr. Saulo* & Average & Extremely high & Average & Extremely high \\
\hline Limoeiro* & Low & Extremely high & Extremely high & Extremely high \\
\hline Rio Itaúnas* & Average & Extremely high & High & Extremely high \\
\hline Ruschi* & Average & Extremely high & Low & Extremely high \\
\hline Casa Branca & Low & Average & Extremely high & High \\
\hline Santa Bárbara & Low & Average & High & High \\
\hline Categories & $\%$ & $\%$ & $\%$ & $\%$ \\
\hline Extremely high $(\%)$ & 6.7 & 33.3 & 13.3 & 33.3 \\
\hline High (\%) & 0 & 26.7 & 20 & 13.3 \\
\hline Average (\%) & 20 & 33.3 & 6.7 & 53.3 \\
\hline Low (\%) & 73.3 & 6.7 & 60 & 0 \\
\hline
\end{tabular}



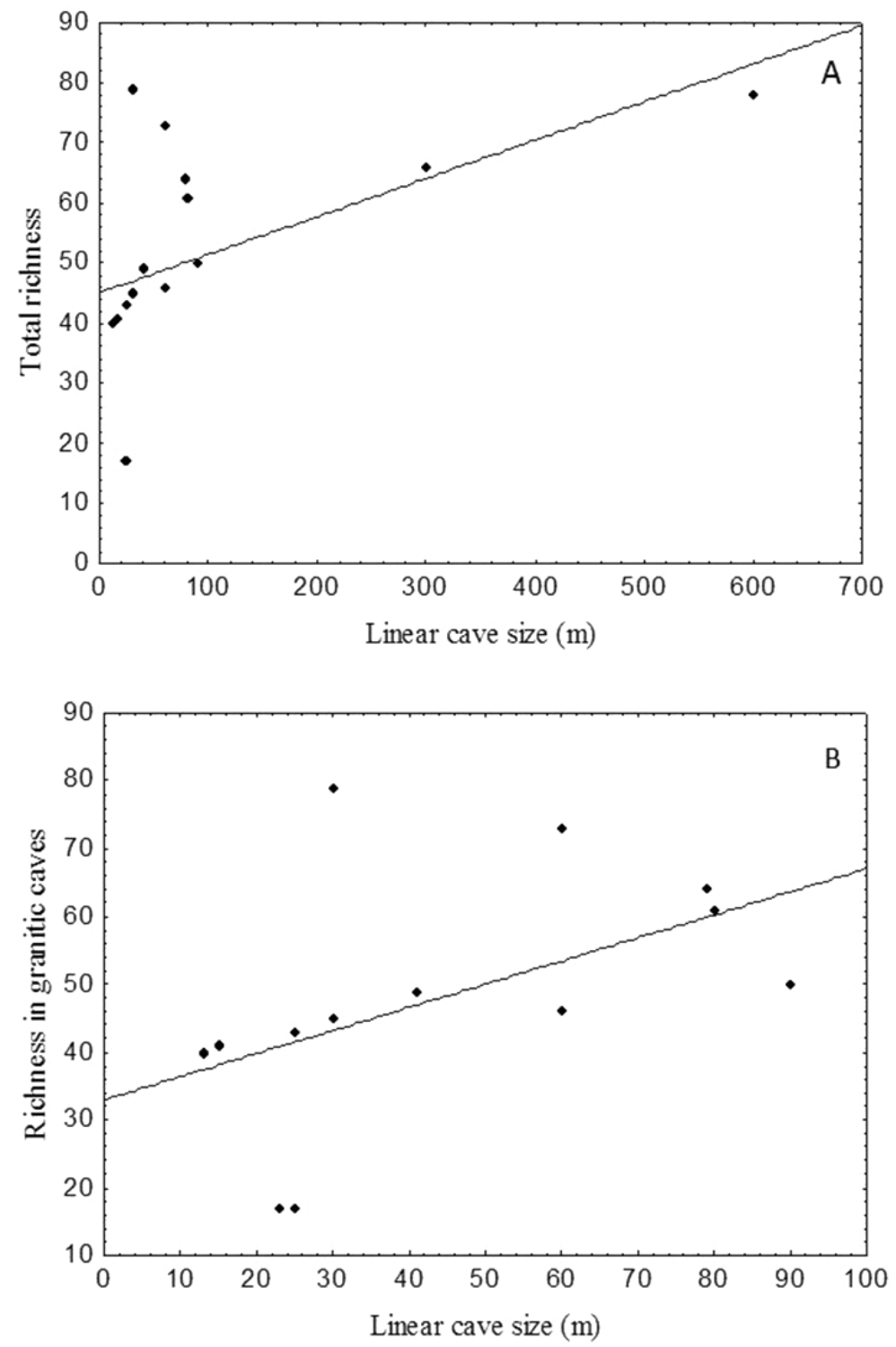

Figure 6. A Significant relationship of the increased richness of collected invertebrates with the increase in size of caves in limestone and granitic rocks and $\mathbf{B}$ no significant relationship without limestone caves in the state of Espírito Santo.

\section{Trophic chain basis}

Qualitatively, the food resources for invertebrates were variable regarding to the type and quantity present in different caves. The most frequent were the plant detritus accumulated near the entrances, bat guano (from hematophagous, insectivorous, frugivo- 
Table 6. Criteria used in defining cave priorities for conservation actions based on 15 caves located at Espírito Santo.

\begin{tabular}{|c|c|c|}
\hline Priority & Degree & Criteria \\
\hline 1 & $\begin{array}{l}\text { Extremely } \\
\text { high }\end{array}$ & $\begin{array}{l}\text { Expected relative occurrence of } 3.76 \text { or more troglobiotic/troglomorphic species } \\
\text { of wide or narrow distribution; total species richness more than } 61 \text { species; } \\
\text { relative richness more than } 0.76 \text { (biological relevance greater than } 6.1 \text { points) } \\
\text { and sum of impact weights more than } 25 \text { points. }\end{array}$ \\
\hline 2 & High & $\begin{array}{l}\text { Expected relative occurrence from } 2.6 \text { to } 3.75 \text { troglobiotic/troglomorphic species } \\
\text { of wide or narrow distribution, total richness between } 41 \text { to } 50 \text { species; relative } \\
\text { richness between } 0.6 \text { and } 0.75 \text { species (biological relevance between } 4.1 \text { and } 6 \\
\text { points) and the sum of impact weights between } 16.6 \text { and } 24.9 \text { points. }\end{array}$ \\
\hline 3 & Average & $\begin{array}{l}\text { Expected relative occurrence from } 1.26 \text { to } 2.5 \text { troglobiotic/troglomorphic species } \\
\text { of wide or narrow distribution; total richness between } 21 \text { and } 40 \text { species; relative } \\
\text { richness between } 0.26 \text { and } 0.5 \text { species (biological relevance between } 2.1 \text { and } 4 \\
\text { points) and the sum of impact weights between } 8.26 \text { and } 16.5 \text { points. }\end{array}$ \\
\hline 4 & Low & $\begin{array}{l}\text { Expected occurrence from } 1.25 \text { or less troglobiotic/troglomorphic species; total } \\
\text { richness less than or equal } 20 \text { species; relative richness less than or equal } 0.25 \\
\text { species (biological relevance less than } 2.1 \text { points) and the sum of impact weights } \\
\text { less than or equal } 8.25 \text { points. }\end{array}$ \\
\hline
\end{tabular}
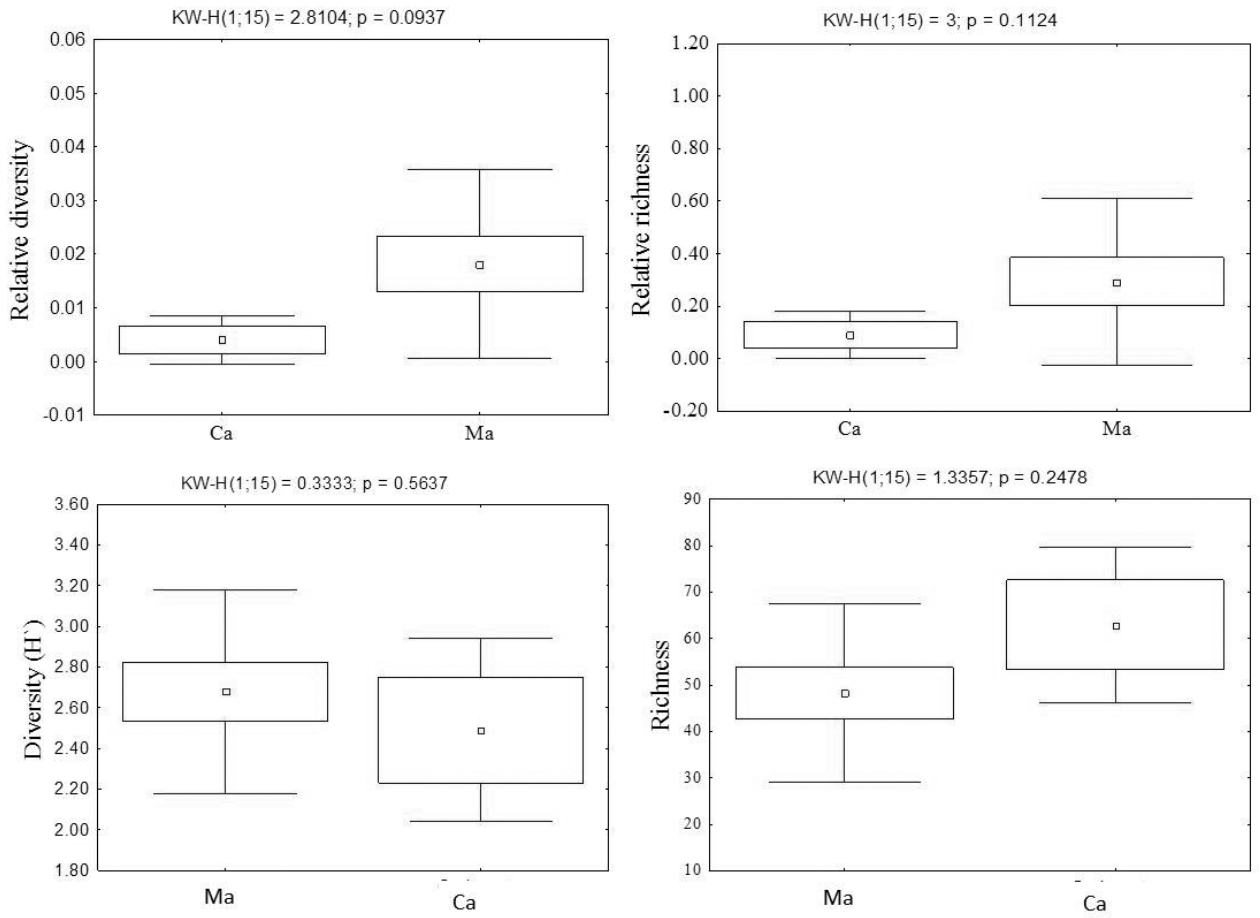

Figure 7. Almost significant differences between the diversity and total and relative richness of invertebrates in caves that develop in carbonate rocks and magma in the state of Espírito Santo. Average, \pm SE, \pm SD. 


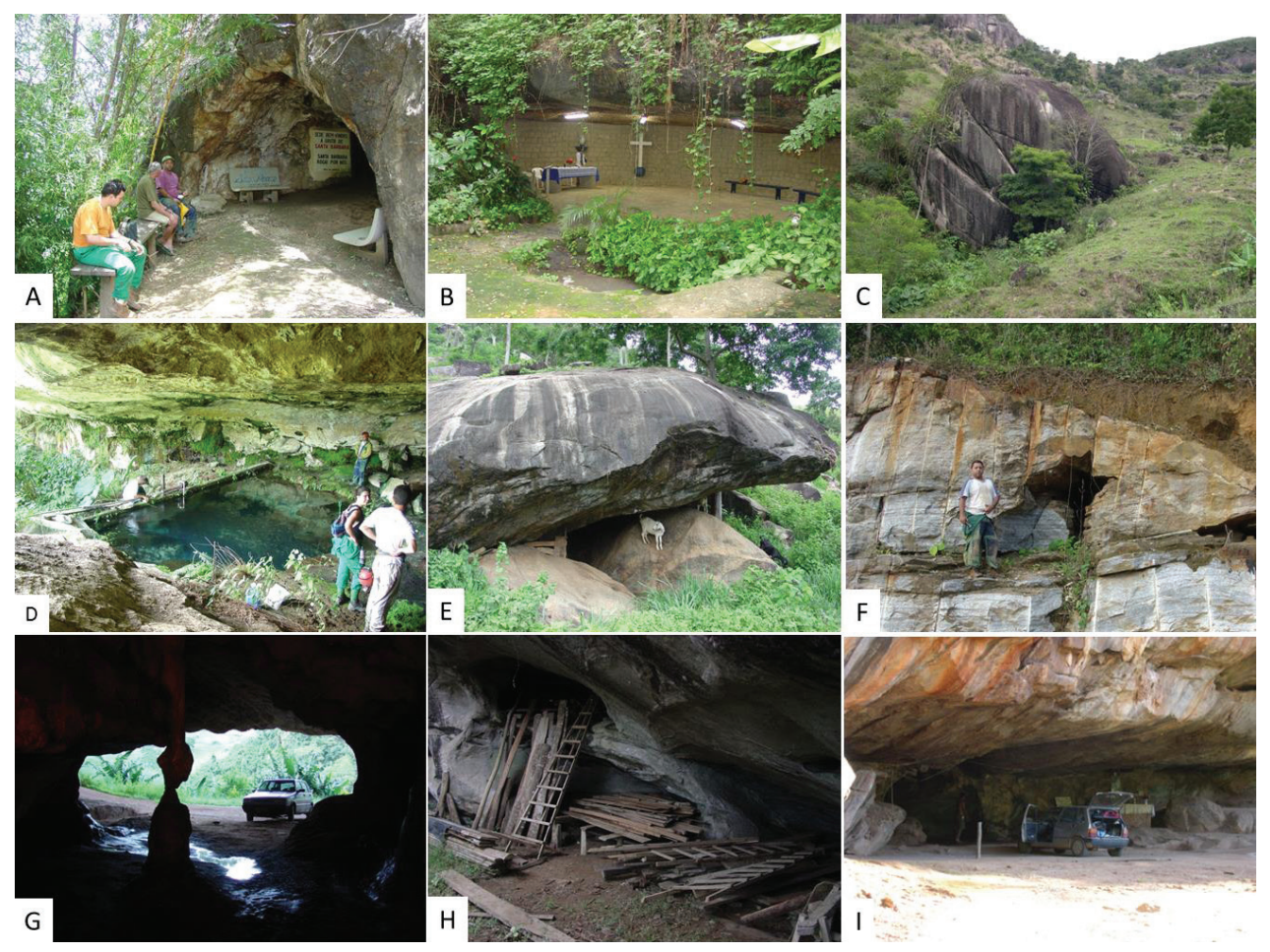

Figure 8. Human alterations in caves of Espírito Santo, Brazil. A religious use in granite cave in Venda Nova dos Imigrantes B transformation of granitic cave into a church in Itaimbé-Itaguassu $\mathbf{C}$ deforestation surrounding cave in Ecoporanga, D drainage exploitation in granite cave near Pedro Canário $\mathbf{E}$ use of cave as goat corral $\mathbf{F}$ road construction destroying cave chambers in Vargem Alta $\mathbf{G}$ and $\mathbf{I}$ Limoeiro cave entrance with religious and tourist use in Conceição de Castelo $\mathbf{H}$ using limestone cave as a timber-yard in Vargem Alta.

rous and carnivorous bats) and roots of external vegetation accessing the cave galleries. The presence of visible food resources for invertebrate fauna was not detected only in the Casa Branca cave. This cave has been transformed into a small church with cemented floors and walls, electric lighting, an altar and wooden benches (Fig. 8B).

\section{Biological relevance, human impact and vulnerability}

Human changes (threats) observed were religious use, tourist use, deforestation of the surroundings, collapse by detonation, trampling, construction, garbage, subterranean drainage exploitation, electrical lighting and degradation of speleothems (Table 4, Fig. 8).

Regarding the biological relevance, 33\% of the caves presented extremely high relevance, 26.7\% high, 33\% average and 6.7\% low (Table 5, Fig. 9). As for the impact weights, $13.3 \%$ of the caves had an extremely high impact degree, $20 \%$ high impact 


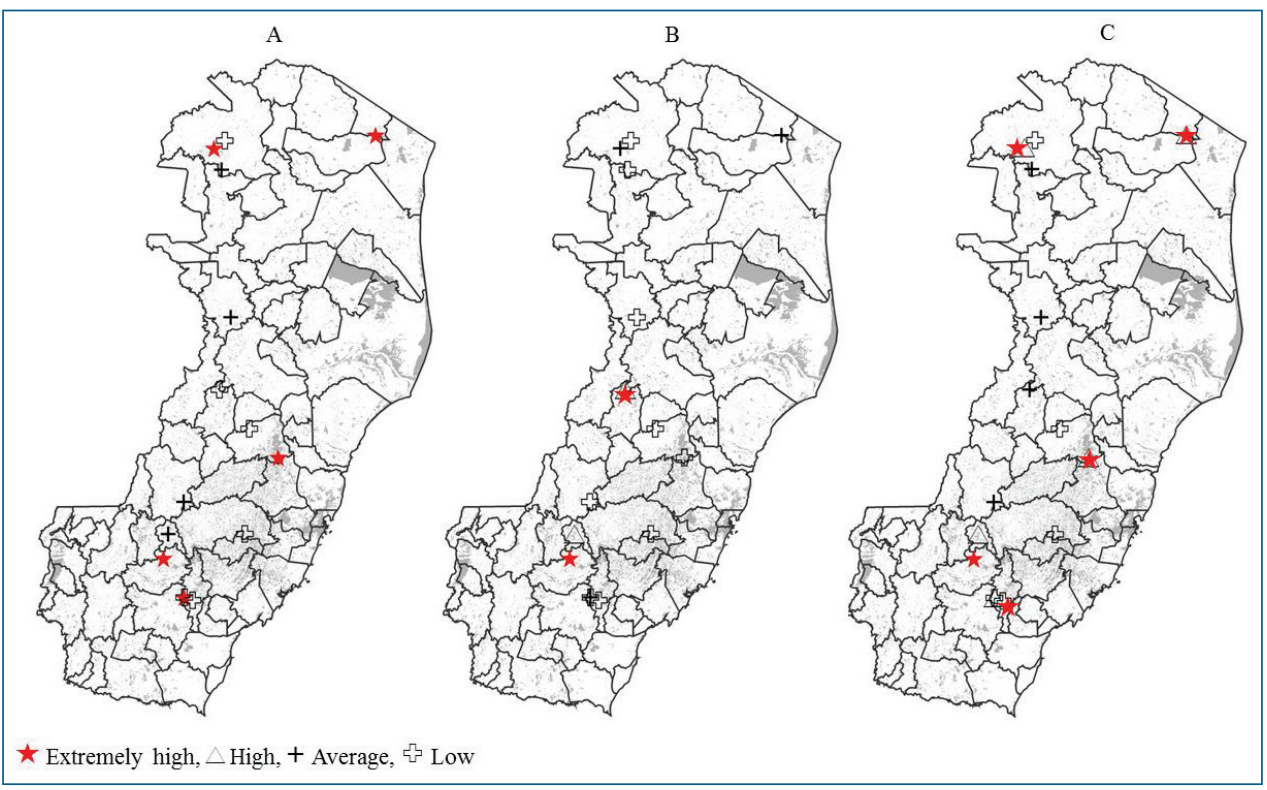

Figure 9. A Distribution of cave biological relevance $\mathbf{B}$ cave impacts category and $\mathbf{C}$ cave fauna vulnerability in the state of Espírito Santo. Gray shading on maps represents remnants of the Atlantic Forest.

degree, $6.7 \%$ average impact degree and $60 \%$ low impact degree. As to vulnerability, $33.3 \%$ showed extremely high vulnerability, $13.3 \%$ high vulnerability, $53.3 \%$ average vulnerability and no cave had a low vulnerability (Table 5, Fig. 9). Table 6 show the summary of the criteria used to assign each cave a conservation priority category.

\section{Suggested conservation actions}

Microbiological research in caves with a suspected presence of Histoplasma sp. was suggested only for the Fazenda Paraíso cave, since two members of the collecting team who had contact with guano presented symptoms and were diagnosed positive for histoplasmosis, including one of the authors (RL Ferreira). The farm owner also reported that a nephew who had visited the cave had a recurring fever (one of the histoplasmosis symptoms), but did not have a confirmed diagnosis.

Studies to define the population size and the real threat status of the troglobiont and endemic species were suggested for five caves. Recovery of cave surroundings that had sustained deforestation activity was suggested for nine caves, with the possibility of using permanent fruit culture (especially cocoa) mixed with native trees, because it can additionally function as an economic and ecological service. Management plans for caves with tourist and/or religious use was suggested for three caves. Maintenance of cave surroundings to preserve external vegetation was suggested for five caves (Private Natural Heritage Reserves-PNHRs). Environmental compensatory measures resulting from completely uncharacteristic cave habitat due to human activities were suggested for one cave (Table 3). 


\section{Suggested karst areas for conservation priorities}

Three karst areas were identified as priorities for conservation action: granitic areas with caves located in the extreme north of Espírito Santo state, in the municipalities of Pedro Canário, and Ecoporanga (24K-395452/7977430), since the caves contain at least three troglomorphic species and face human modifications such as deforestation, agriculture, livestock and groundwater water exploitation. Another area is the granitic mountain rocks with caves in Santa Teresa municipality (24K-339370/7791692), since it presents at least two troglomorphic species and faces human impact, such as deforestation, agriculture, livestock, subterranean drainage alteration. The third area comprises the carbonate area with caves in Castelo, Vargem Alta and Cachoeiro do Itapemirim municipalities (24K-285168/7711062), with at least five species of troglobiont/troglomorphic species and faces human alterations, such as religious and tourism uses, deforestation, mining activities and road construction.

\section{Discussion}

\section{Cave invertebrate community structure}

The composition of the invertebrate cave fauna evaluated in this study, at least to the family level, is very similar to that registered for other caves in the Brazilian Atlantic Forest (Pinto da Rocha 1995, Trajano 2000, Souza-Silva and Ferreira 2009, SouzaSilva et al. 2011c, d). However, the cave fauna collection methodology employed in Brazil until the 1990's apparently has not resulted in an effective sampling of the fauna (due to the small number of species recorded) (Souza-Silva et al. 2011a and c). Furthermore, works carried out to the 1980's did not have any information regarding the abundance or diversity, and those related to fauna richness are underestimated.

It is important to consider that the work was developed almost two decades ago, in a scenario where the main goal was to conduct primary surveys. Currently, it is essential to include more consistent ecological analyses when the goal is to characterize a subterranean community (Souza-Silva et al. 2011c). Thus, our comparisons were based on Souza-Silva et al. (2011a, 2015) who presented a comprehensive characterization of the invertebrate community structure and threats in the Brazilian Atlantic Forest caves.

The average diversity presented by Souza-Silva et al. (2011a) for the fauna of 91 caves in the Brazilian Atlantic Forest (average $=2.27$, sd $=0.63$ ) is similar to the average found for the 15 caves sampled in the state of Espírito Santo (Table 3). However, the average richness presented by the same 91 caves $($ mean $=43.20 \mathrm{sd}=22.30)$ was slightly lower than that found in this present study (Table 3 ). The $\beta$ diversity (turnover) of the 15 caves of this study were slightly higher than the range presented by Souza-Silva et al. (2011a) ( $\beta$ diversity $=60.19$ to 45.58 ).

Different caves may have considerably different richness and diversity values, even if using similar sampling efforts. According to Ferreira (2005) and Souza-Silva (2008), these differences may be due to several factors, such as the linear extension of the caves, their trophic conditions and the degree of their human interventions. 
The $\beta$-diversity values and similarities found show large differences in the community composition of 15 caves and reveal that nearby caves do not necessarily have similar communities. As well as the influence of the type of rock in which the cave is located, variations in the physical (microhabitat availability, humidity, etc.) and trophic structures may lead to differences in the composition and distribution of cave invertebrate communities (Christman and Culver 2001, Schneider et al. 2011, SouzaSilva et al. $2011 \mathrm{a}, \mathrm{b})$. Thus, the relationship of increased total richness with increasing cave size can probably be related to the increased availability of microhabitats and food resources for invertebrate fauna (Souza-Silva et al. 2011a). Bat communities, producing large guano deposits, are richer and more abundant in larger caves (Brunet and Medellin 2001). Large deposits, in turn, provide food and microhabitats for a greater number of invertebrate species (Pellegrini and Ferreira 2013). It is noteworthy that the productivity in cave environments is an important predictor of increased richness in invertebrate communities (Culver et al. 2006). However, the use of the cave extension as a parameter to predict the invertebrate richness depends directly on the lithology to which the cave is associated (Souza-Silva et al. 2011a). In this study, limestone caves working as an outlier in the general relationship displayed increasing richness with an increase in linear extension, because there is no significant relationship when the Archimides Pansini cave $(300 \mathrm{~m})$ and Limoeiro cave $(600 \mathrm{~m})$ were removed from the analysis. Souza-Silva et al. (2011a) also observed this lack of correlation between cave extension and richness in granite caves in the Atlantic forest as a whole.

\section{Environmental impacts on cave fauna}

The Casa Branca cave, located in Itaimbé municipality, showed the least similarity when compared to the other caves. This may be due to the impact of the construction of a church within the cave (Fig. 7B). This prevents the presence of species characteristic of natural cave environments and favors the dominance of opportunistic species tolerant of altered environments.

Troglobiont invertebrates can demonstrate tolerance to certain types of changes which may even occur in partially impacted caves (Simon and Buikema 1997). However, there are certain effects that lead to a complete substitution of the groups that would be expected to be found in such caves. It is important to emphasize that similarity comparisons do not necessarily provide indications of direct impacts: the only effective way to detect the existence of alterations would be to have had the opportunity to know the fauna of these caves before these alterations could have occurred. Under these circumstances, one should make use of other tools to compare the structure and faunal composition of the caves in order to understand the effects of the observed changes. While not the best "tools", such comparisons allow the evaluation, even crudely, of how some impacts may alter the conditions of pristine subterranean ecosystems. 


\section{Relevance of cave fauna for conservation}

When comparing the richness of troglobiont species found in this study with other karst regions in Brazil and around the world (Culver and Sket 2000; Culver and Pipan 2013; Souza-Silva et al. 2011a, 2015), it can be perceived that the caves sampled to date in the state of Espírito Santo are relatively poor, and cannot be considered hotspots for troglobiont species biodiversity (Fig. 10 and Table 2). However, due to their rarity, the independent phylogenetic history and vulnerability to extinction of the troglobiont species, they deserve special conservation measures. Furthermore, the trogloxene and troglophile invertebrate communities appear to be relatively richer in species compared to other caves in Brazil (Souza-Silva et al. 2011a).

\section{Final considerations}

Facing the conditions found in the sampled caves up to now, we recommend emergency attention regarding protective measures, the management and conservation of caves of extremely high and high biological importance, with high impact degree and extremely high vulnerability facing the impacts. It is very important to intensify speleological studies and faunistic surveys, since some areas in the Atlantic Forest in Espírito Santo with the potential for cave occurrence have not been intensively studied (Castelo, Vargem Alta, Nova Venécia, municipalities). Reforestation of at least 250 meters of the cave surroundings that have surrounding pastures, using agroforestry models and management plans and/or ecological restoration of the caves that have received human impacts that come from tourism, religious use and implementation of information and awareness plans for users, with the accompaniment of environmental inspectors at times of mass celebrations and holy days, should be undertaken. Finally it is important to stimulate the creation of Private Natural Heritage Reserves (PNHRs) in the surroundings of caves with troglobiont species, preserved natural vegetation or that under recuperation.

\section{Acknowledgments}

Critical Ecossystem Partnership Fund (CEPF), Aliança for the conservation of the Mata Atlântica, Conservação Internacional (CI), IBAMA-CECAV, S.O.S. Mata Atlântica, Empresa de Assistência Técnica e Extensão Rural (Emater, MG), Estação Biologia Marinha Ruschi, Instituto de Defesa Agropecuária e Florestal (IDAF, MG) ES. We thank Leopoldo Ferreira, Erika Linzi Taylor and Augusto Cezar Francisco Alves for their support in the fieldwork. R. Ferreira is grateful to the Fundação de Amparo à Pesquisa do Estado de Minas Gerais (FAPEMIG) for funding support and to the National Council of Technological and Scientific Development (CNPq) for the research grant (process No. 304682/2014-4). License number: 0182/2004 CGFAU/LIC and IBAMA 02001.005461/2004 


\section{References}

Aguiar AP, Chiarello AG, Mendes SL, Matos EN (2005) Os Corredores Central e da Serra do Mar na Mata Atlântica Brasileira. In: Galindo-Leal C, Câmara IG (Eds) Mata Atlântica; biodiversidade, ameaças e perspectivas, Belo Horizonte; Centro de Ciências Aplicadas e Biodiversidade, 119-132.

Bodmer RE, Eisenberg JF, Redford HK (1997) Hunting and the Likelihood of Extinction of Amazonian Mammals. Conservation Biology 2(11): 460-466. doi: 10.1046/j.15231739.1997.96022.x

Brown JRKS (1972) Maximizing daily butterfly counts. Journal of the Lepidopterists' Society 26(3): 183-196.

Brunet AK, Medelin RA (2001) The species-area relationship in bat assemblages of tropical caves. Journal of Mammalogy 82(4): 1114-1122. doi: 10.1644/1545-1542(2001)082<1114:TS ARIB > 2.0.CO;2

CEBS (2015) Center of studies on subterranean biology from Federal University of Lavras, Minas Gerais, Brazil. http://www.biologiasubterranea.com.br

Chiarello AG (1995) Density and habitat use of primates at an Atlantic forest reserve of southeastern Brazil. Revista Brasileira de Biologia 55(1): 105-110.

Christman MC, Culver DC (2001) The relationship between cave biodiversity and available habitat. Journal of Biogeography 3(28): 367-380. doi: 10.1046/j.1365-2699.2001.00549.x

Costa-Lima A, Costa-Leite I (1953) Um novo grilo cavernicola (Orthoptera, Gryllidae, Phalangopsidae). Anais Academia Brasileira de Ciências 25: 169-170.

Culver DC, Deharveng L, Bedos A, Lewis JJ, Madden M, Reddell JR, Sket B, Trontelj P, White D (2006) The mid-latitude biodiversity ridge in terrestrial cave fauna. Ecography 29: 120-128. doi: 10.1111/j.2005.0906-7590.04435.x

Culver DC, Pipan T (2009) The biology of caves and other subterranean habitats. Library of Congress Cataloging in Publication Data, Oxford University Press, Oxford, 254 pp.

Culver DC, Pipan T (2013) Subterranean Ecosystems. In: Levin SA (Ed.) Encyclopedia of Biodiversity. Academic Press, Waltham, 49-62. doi: 10.1016/b978-0-12-384719-5.00224-0

Culver DC, Sket B (2000) Hotspots of Subterranean Biodiversity in Caves and Wells. Journal of Cave and Karst Studies 62(1): 11-17.

Dessen EMB, Eston VR, Silva MS, Temperini-Beck MT, Trajano E (1980) Levantamento preliminar da fauna de cavernas de algumas regióes do Brasil. Ciência e Cultura 32(6): $714-725$.

Ferreira RL, Horta LCS (2001) Natural and human impacts on invertebrate communities in Brazilian caves. Revista Brasileira de Biologia 61(1): 7-17. doi: 10.1590/S003471082001000100003

Ferreira RL (2005) A vida subterrânea nos campos ferruginosos O Carste 3: 106-115.

Galindo LC, Câmara IG (2005) State of the hotspots: Mata Atlântica: uma síntese. In: Mata Atlântica; biodversidade, ameaças e perspectivas. In: Galindo-Leal C, Câmara, IG (Eds) Belo Horizonte. Centro de Ciências Aplicadas e Biodiversidade, 471 pp.

Hammer O, Harper DAT, Ryan PD (2001) PAST: Paleontological Statistics Software Package for Education and Data Analysis. Palaeontologia Electronica 4(1): 1-9. 
Isaac N JB, Cowlishaw G (2004) How species respond to multiple extinction threats. Proceedings of the Royal Society B: Biological Sciences 271: 1135-1141. doi: 10.1098/ rspb.2004.2724

MacArthur RH, Wilson EO (1967) The Theory of Island Biogeography. Princeton University Press, Princeton, 463 pp.

Maciel MAO (2000) Sistema Nacional de Unidades de Conservação da Natureza - SNUC. http://www.planalto.gov.br/ccivil_03/leis/19985.htm

Magurran AE (2004) Ecological Diversity and Its Measurement. Cromm Helm, London, 179 pp.

McKinney ML (1997) Extinction vulnerability and selectivity: Combining ecological and paleontological views. Annual Review of Ecology and Systematics 28: 495-516. doi: 10.1146/ annurev.ecolsys.28.1.495

Parise M, Gunn J (Eds) (2007) Natural and anthropogenic hazards in karst areas: recognition, analysis and mitigation. Geological Society, London, Special Publications, 279 pp.

Passamani M, Mendes SL, Chiarello AG (2000) Non Volant mammals of the Estação Biológica de Santa Lúcia and adjacent areas of Santa Teresa, Espírito Santo, Brasil. Boletim do Museu de Biologia Mello Leitão (Nova Série) 11/12: 201-214.

Peixoto AL, Gentry A (1990) Diversidade e composição florística da mata de tabuleiro na Reserva Florestal de Linhares (Espírito Santo, Brasil). Revista Brasileira de Botânica 13: 19-25.

Pellegrini TG, Ferreira RL (2013) Structure and interactions in a cave guano-soil continuum community. European Journal of Soil of Biology 57: 19-26.

Pimm SL, Jones HL, Diamond J (1988) On the risk of extinction. American Naturalist 132: 757-785. doi: 10.1086/284889

Pinto-da-Rocha R (1995) Sinopse da fauna cavernícola do Brasil. Papéis Avulsos de Zoologia 39(6): 61-173.

Prous X, Ferreira RL, Martins RP (2004) Ecotone delimitation: epigean-hypogean transition in cave ecosystems. Austral Ecology 29: 374-382. doi: 10.1111/j.1442-9993.2004.01373.x

Prous X, Ferreira RL, Jacobi CM (2015) The entrance as a complex ecotone in a Neotropical cave. International Journal of Speleology 44(2): 177-198. doi: 10.5038/1827-806X.44.2.7

Purvis A, Gittleman JL, Cowlishaw G, Mace GM (2000) Predicting extinction risk in declining species. Proceedings: Biological Sciences 1456(267): 1947-1952. doi: 10.1098/ rspb.2000.1234

Ribeiro AM (1907) Uma novidade ichthyologica. Kosmos 4(1): 21-22.

Ribeiro MC, Metzger JP, Martensena AC, Ponzoni FJ, Hirota MM (2009) The Brazilian Atlantic Forest: How much is left, and how is the remaining forest distributed? Implications for conservation. Biological Conservation 142: 1141-1153. doi: 10.1016/j.biocon.2009.02.021

Ruschi A (1952) Morcegos do estado do Espírito Santo IX. Boletim do Museu de Biologia Mello-Leitão, Série Zoologia 9(A): 1-92.

Schneider K, Christman MC, Fagan WF (2011) The influence of resource subsidies on cave invertebrates: results from an ecosystem-level manipulation experiment. Ecology 92(3): 765-776. doi: 10.1890/10-0157.1

Simóes MH, Souza-Silva M, Ferreira RL (2014) Cave invertebrates in northwestern Minas Gerais state, Brazil: endemism, threats and conservation priorities. Acta Carsologica 43(1): 159-174. doi: 10.3986/ac.v43i1.577 
Simon KS, Buikema AL (1997) Effects of Organic Pollution on an Appalachian Cave: Changes in Macroinvertebrate Populations and Food Supplies. American Midland Naturalist 138: 387-401. doi: 10.2307/2426830

Sket B (2008) Can we agree on an ecological classification of subterranean animals? Journal of Natural History 21-22(42): 1549-1563. doi: 10.1080/00222930801995762

SOS Mata Atlântica and Instituto Nacional de Pesquisas Espaciais (2014) Atlas dos remanescentes florestais da mata atlântica período 2008-2010. http://www.sosma.org.br

Souza MFVR, Ferreira RL (2011) A new troglobitic Eukoenenia (Palpigradi: Eukoeneniidae) from Brazil. Journal of Arachnology 39(1): 185-188. doi: 10.1636/Ha10-43.1

Souza-Silva M, Ferreira RL (2009) Estrutura das comunidades de invertebrados em cinco cavernas insulares e intertidais na costa brasileira. Espeleo-Tema 20(1/2): 25-36.

Souza-Silva M, Martins RP, Ferreira RL (2011a) Cave lithology determining the structure of the invertebrate communities in the Brazilian Atlantic Rain Forest. Biodiversity and Conservation 8(20): 1713-1729. doi: 10.1007/s10531-011-0057-5

Souza-Silva M, Martins RP, Ferreira RL (2011b) Trophic Dynamics in a Neotropical Limestone Cave. Subterranean Biology 9: 127-138. doi: 10.3897/subtbiol.9.2515

Souza-Silva M, Martins RP, Ferreira RL (2015) Cave Conservation Priority Index to Adopt a Rapid Protection Strategy: A Case Study in Brazilian Atlantic Rain Forest. Environmental Management 55: 279-295. doi: 10.1007/s00267-014-0414-8

Souza-Silva M, Nicolau JC, Ferreira RL (2011c) Comunidades de invertebrados terrestres de três cavernas quartzíticas no Vale do Mandembe, Luminárias, MG. Espeleo-Tema 1(22): 79-91.

Tercafs R (1992) Conservation: cave biota. In: Gunn J (Ed.) Encyclopedia of caves and karst science. New York, 500-503.

Thomas WW, Carvalho AMV, Amorim AMA, Garisnon J, Arbeláez AL (1998) Plant endemism in two forest in southern Bahia, Brazil. Biodiversity and Conservation 7: 311-322. doi: 10.1023/A:1008825627656

Thomaz LD, Monteiro R (1997) Composição florística da Mata Atlântica de encosta da Estação Biológica de Santa Lúcia, município de Santa Teresa - ES. Boletim do Museu de Biologia Mello Leitão 7: 1-86.

Trajano E (2000) Cave faunas in the Atlantic tropical rain forest: composition, ecology and conservation. Biotropica 32: 882-893. doi: 10.1111/j.1744-7429.2000.tb00626.x

Webb JK, Brook BW, Shine R (2002) What makes a species vulnerable to extinction? Comparative life-history traits of two sympatric snakes Ecological Research 17: 59-67. doi: 10.1046/j.1440-1703.2002.00463.x

Whitmore TC, Sayer JA (1992) Tropical deforestation and species extinction. Chapman and Hall, London, 153 pp.

Zar JH (1984) Biostatistical analysis. Prentice-Hall International Editions, New Jersey, 718 pp. 